\title{
Impacto da Prática Habitual de Atividade Física no Perfil Lipídico de Adultos
}

\section{artigo original}

\section{Dartagnan Pinto Guedes LEANDRO A.V. Verde Gonçalves}

Universidade Estadual de Londrina, PR.

O objetivo do estudo foi analisar o impacto da prática habitual de atividade física, mediante informações reunidas por intermédio do International Physical Activity Questionnaire (IPAQ), no perfil lipídico em amostra representativa de sujeitos adultos. A amostra foi constituída por 361 sujeitos (196 mulheres e 165 homens) com idades entre 20 e 60 anos. As concentrações de lipídeos plasmáticos foram estabelecidas mediante procedimentos laboratoriais. Os achados revelaram que, em ambos os sexos, os níveis plasmáticos de HDL-colesterol e VLDL-colesterol não apresentaram diferenças significativas entre os grupos de prática habitual de atividade física. Os valores de triglicerídeos, colesterol sérico total e LDL-colesterol foram significativamente diferentes em mulheres e homens, porém o comportamento de variação foi diferente nos dois sexos. As informações da análise de regressão logística indicaram que as odds ratios para menores valores de lipídeos plasmáticos foram significativas somente nos sujeitos classificados como muito-ativos em comparação com os sedentários. Como conclusão, os resultados sugerem que mais elevada prática habitual de atividade física determinada pelo IPAQ apresenta significativo impacto no perfil lipídico em adultos de ambos os sexos, independentemente da idade e das variações do peso corporal. (Arq Bras Endocrinol Metab 2007;51/1:72-78)

Descritores: IPAQ; Estilo de vida; Sedentarismo; Exercício físico; Colesterol; Triglicerídeos

\section{ABSTRACT}

Impact of the Habitual Physical Activity on Lipid Profile in Adults. The present study was designed to analyze the impact of the habitual physical activity determined by International Physical Activity Questionnaire (IPAQ) on lipid profile in representative sample of adults. A sample of 361 subjects (196 women and 165 men) aged 20-60 years old was included in the study. Plasma lipid concentrations were measured by standard procedures. The results showed that HDL-cholesterol and VLDL-cholesterol levels were not significantly different across physical activity groups in both sexes. Total cholesterol, LDL-cholesterol and triglycerides were significantly different across physical activity groups in women and men, but the pattern of variation was different between genders. The results from logistic regression analysis indicated that the odds ratios for low plasma lipid levels was significant only in subjects classified as very active compared with sedentary. In conclusion, the results suggest that increased habitual physical activity determined by IPAQ present significant impact in the plasma lipid profile in adults of both sexes, independently of the age and of the variations of the body weight. (Arq Bras Endocrinol Metab 2007;51/1:72-78)

Recebido em 06/04/06 Aceito em 14/08/06
Keywords: IPAQ; Lifestyle; Sedentarism; Physical exercise; Cholesterol; Triglycerides 
$\mathrm{E}$ M ESTUdOS ENVOLVENDO sujeitos adultos, comprova-se que o estilo de vida sedentário é um comportamento claramente identificado com perfil lipídico desfavorável (1-4). Em tese, assume-se que a associação observada entre a prática insuficiente de atividade física e as dislipidemias possa explicar parcialmente o menor risco predisponente ao aparecimento e ao desenvolvimento de doenças cardiovasculares em indivíduos mais fisicamente ativos (5-7).

Em vista disso, a monitoração quanto à prática habitual de atividade física tem recebido grande notoriedade no campo da saúde, não apenas por sua ação isolada na prevenção e no controle das doenças cardiovasculares (8-11), mas também por induzir a alterações desejáveis nos níveis de lipídeos plasmáticos (12-15). Diante desta perspectiva, o impacto da prática habitual de atividade física no perfil lipídico pode não se confirmar com o mesmo potencial dependendo da natureza e das especificações do método empregado na coleta das informações equivalentes à prática habitual de atividade física.

Neste particular, apesar da disponibilidade de vários métodos direcionados ao acompanhamento de indicadores relacionados à prática habitual de atividade física (16), em razão das facilidades de aplicação e de sua inocuidade, os questionários auto-administrados acerca das atividades desempenhadas no cotidiano têm-se definido como uma opção freqüentemente recomendada para esse tipo de análise $(17,18)$.

Com relação às opções de questionários disponíveis, apoiado em evidências quanto ao atendimento dos critérios de validação e de reprodutibilidade, mais recentemente o Questionário Internacional de Atividade Física (International Physical Activity Questionnaire - IPAQ) tem recebido atenção especial. Em sendo assim, o objetivo do presente estudo foi analisar o impacto da prática habitual de atividade física, mediante informações reunidas por intermédio do IPAQ, no perfil lipídico de adultos de ambos os sexos.

\section{CASUÍSTICA E MÉTOdOS}

Para a elaboração do estudo foram selecionados sujeitos de ambos os sexos que procuraram os programas de exercícios físicos oferecidos pela CEN - Clínica de Endocrinologia e Nutrição, localizado na cidade de Londrina, Paraná, durante o período de junho a dezembro de 2004. A inclusão dos sujeitos na amostra ocorreu por desejo de participar do estudo e que atendia aos cinco critérios básicos: (a) ter entre 20 e 60 anos de idade; (b) ser não-fumante nos últimos 5 anos; (c) não estar sendo submetido a dietas especiais; (d) não utilizar medicamentos de uso contínuo; e (d) não ser portador de doenças cardiovasculares ou metabólicas. Em assim sendo, a amostra definitiva do estudo foi constituída por 361 sujeitos (196 mulheres e 165 homens).

Os procedimentos empregados no estudo foram aprovados pelo Comitê de Ética em Pesquisa da Universidade Estadual de Londrina e acompanharam normas da Resolução 196/96 do Conselho Nacional de Saúde sobre pesquisa envolvendo seres humanos. Após leitura individual e receberem eventuais esclarecimentos quanto aos objetivos e aos procedimentos do estudo, os sujeitos assinaram termo de consentimento informado previamente aprovado pelo Comitê de Ética.

No campo antropométrico foram realizadas medidas de estatura e de peso corporal, a partir de padronizações sugeridas por Gordon e cols. (19). Estabeleceu-se, também, cálculo do índice de massa corporal (IMC) considerando a razão entre as medidas do peso corporal e do quadrado da estatura $\left(\mathrm{kg} / \mathrm{m}^{2}\right)$.

As dosagens dos lipídeos plasmáticos foram realizadas mediante coleta de amostras de $10 \mathrm{ml}$ de sangue venoso na prega do cotovelo, após período de 10-12 h em jejum, entre 07:00 e 08:00h da manhã. O soro foi imediatamente separado por centrifugação, sendo determinados os teores de triglicerídeos (TG), colesterol sérico total (CT) e frações, lipoproteínas de alta densidade (HDL-C), de baixa densidade (LDL-C) e de muito-baixa densidade (VLDL-C). Determinou-se o CT pelo método enzimático colesterol oxidase/peroxidase em aparelho espectofotômetro. O HDL-C foi medido pelo método reativo precipitante, e o LDL-C e o VLDL-C foram calculados pela fórmula de Friedewald (20). Os TG foram determinados pelo método enzimático glicerol. Além dos valores absolutos de cada componente plasmático, consideraram-se as relações CT/HDL-C e LDL-C/HDL-C. Os valores de referência empregados para definir um perfil lipídico-lipoprotêico de risco aterogênico acompanharam proposta apresentada mediante as III Diretrizes Brasileiras sobre Dislipidemias (2l): CT $\geq 240 \mathrm{mg} / \mathrm{dl}$, LDL-C $\geq 160 \mathrm{mg} / \mathrm{dl}$, HDL-C $\leq 40 \mathrm{mg} / \mathrm{dl} \mathrm{e} \mathrm{TG} \geq 200 \mathrm{mg} / \mathrm{dl}$.

Informações equivalentes à prática habitual de atividade física foram obtidas mediante o IPAQ, proposto pelo Grupo Internacional para Consenso em Medidas da Atividade Física, constituído sob a chancela da Organização Mundial da Saúde, com representantes de 25 países, inclusive do Brasil (22). Originalmente, o IPAQ é apresentado em diferentes idiomas, inclusive em língua portuguesa, o que dispensou a necessidade de sua tradução. Optou-se por analisar o questionário de auto-administração em seu formato curto, versão 8 , tendo como referência a última semana. As questões indagam quanto à freqüência (dias/semana) e ao tempo (minutos/dia) despendido na execução de caminhadas e de atividades envolvendo esforços físicos de intensidades moderada e vigorosa. A aplicação do IPAQ foi realizada individualmente. Para tanto, os participantes do estudo recebiam o questionário com instruções e recomendações para o seu preenchimento, não sendo estabelecido limite de tempo para o seu preenchimento e as eventuais dúvidas manifestadas pelos sujeitos eram prontamente esclarecidas pelo 
profissional que acompanhava a coleta de dados. Para categorização da prática habitual de atividade física recorreu-se ao consenso proposto pelo Centro de Estudos do Laboratório de Aptidão Física de São Caetano do Sul (23), considerando quatro estratos:

Muito-Ativo: $\geq 30$ minutos/sessão de atividades vigorosas $\geq 5$ dias/semana; e/ou $\geq 20$ minutos/sessão de atividades vigorosas $\geq 3$ dias/semana somadas a $\geq 30$ minutos/sessão de atividades moderadas ou caminhadas $\geq 5$ dias/semana;

Ativo: $\geq 20$ minutos/sessão de atividades vigorosas $\geq$ 3 dias/semana; e/ou $\geq 30$ minutos/sessão de atividades moderadas ou caminhadas $\geq 5$ dias/semana; e/ou $\geq 150$ minutos/semana de qualquer das atividades somadas (vigorosa + moderada + caminhada);

Irregularmente Ativo: < 150 e > 10 minutos/semana de qualquer das atividades somadas (vigorosa + moderada + caminhada); e

Sedentário: $\leq 10$ minutos/semana de qualquer das atividades somadas (vigorosa + moderada + caminhada).

O tratamento estatístico das informações foi realizado mediante o pacote computadorizado Statistical Package for the Social Sciences (SPSS), versão 13.0. Para análise das variáveis contínuas recorreu-se aos procedimentos da estatística descritiva e, posteriormente, para identificação de eventuais diferenças entre os sexos, ao teste " $t$ " de Student. Comparações entre os valores equivalentes aos lipídeos plasmáticos dos sujeitos classificados em cada um dos quatro estratos de prática habitual de atividade física foram realizadas mediante análise de co-variância. Na tentativa de controlar os efeitos adicionais quanto ao envelhecimento e ao sobrepeso no impacto da atividade física sobre o perfil lipídico, as informações associadas à idade e ao IMC foram consideradas como co-variáveis na análise estatística. Valores de odds ratio (OR), estabelecidos por intermédio da análise de regressão logística binária, também controlando informações associadas à idade e ao IMC, foram utilizados para estabelecer estimativas quanto ao risco relativo de apresentar perfil lipídico de risco aterogênico em função da categorização em estratos decrescentes de prática habitual de atividade física. Adotou-se intervalo de confiança de 95\%.

\section{RESULTADOS}

Informações estatísticas com relação às variáveis analisadas encontram-se na tabela 1. Quanto ao IMC, os valores médios encontrados apontam diferenças estatísticas favoráveis aos homens. Aproximadamente $38 \%$ da amostra analisada apresentaram IMC $\geq 28$ $\mathrm{kg} / \mathrm{m}^{2}$, o que oferece indícios de sobrepeso. No que se refere ao perfil lipídico, os teores de CT, a fração HDL-C e a relação CT/HDL-C mostram semelhanças entre os sexos. Porém, homens demonstram valores médios equivalentes às concentrações séricas de LDL-C, VLDL-C, TG e a relação LDL-C/HDL-C estatisticamente mais elevados. Ao recorrer aos valores de referência sugeridos mediante as III Diretrizes Brasileiras sobre Dislipidemias (21), verifica-se que o perfil lipídico, associado ao aumento do risco de doenças cardiovasculares, foi comum em aproximadamente $21 \%$ da amostra estudada: $21,8 \%$ apresentaram dosagem de CT $\geq 240 \mathrm{mg} / \mathrm{dl} ; 17,7 \%$ LDL-C $\geq 160$ $\mathrm{mg} / \mathrm{dl} ; 22,7 \%$ HDL-C $\leq 40 \mathrm{mg} / \mathrm{dl}$ e $22,3 \%$ concentrações de $\mathrm{TG} \geq 200 \mathrm{mg} / \mathrm{dl}$.

Resultados equivalentes à análise de co-variância, controlando estatisticamente as informações associadas à idade e ao IMC, são mostrados na tabela 2. $\mathrm{O}$ perfil lipídico dos sujeitos analisados no estudo apresenta diferenças de acordo com os estratos de prática habitual de atividade física sugeridos pelo IPAQ. Aqueles sujeitos de ambos os sexos que relataram ser mais ativos fisicamente demonstram teores de TG, CT e da fração LDL-C, além das relações CT/HDL-C e LDL-C/HDL-C, mais favoráveis. Verifica-se também que o comportamento de variação dos valores observados é diferente nos dois sexos. Enquanto nos homens as diferenças mais acentuadas são constatadas entre o estrato dos muito-ativos em comparação com seus pares irregularmente ativos e sedentários, nas mulheres esse fenômeno é observado entre o estrato

Tabela 1. Comparação dos dados clínicos e laboratoriais entre os sujeitos de ambos os sexos analisados no estudo.

\begin{tabular}{lccc}
\hline & $\begin{array}{c}\text { Mulheres } \\
(\mathbf{n = 1 9 6 )}\end{array}$ & $\begin{array}{c}\text { Homens } \\
(\mathbf{n = 1 6 5 )}\end{array}$ & $\mathbf{p}$ \\
\hline Idade (anos) & $40,41 \pm 10,43$ & $39,13 \pm 9,37$ & $\mathrm{~ns}$ \\
IMC $\left(\mathrm{kg} / \mathrm{m}^{2}\right)$ & $29,89 \pm 5,51$ & $31,37 \pm 5,21$ & 0,009 \\
CT $(\mathrm{mg} / \mathrm{dl})$ & $208,87 \pm 39,80$ & $203,02 \pm 34,97$ & $\mathrm{~ns}$ \\
\hline HDL-C $(\mathrm{mg} / \mathrm{dl})$ & $46,67 \pm 14,42$ & $44,43 \pm 9,33$ & $\mathrm{~ns}$ \\
LDL-C $(\mathrm{mg} / \mathrm{dl})$ & $116,50 \pm 37,78$ & $127,08 \pm 39,10$ & 0,010 \\
\hline VLDL-C $(\mathrm{mg} / \mathrm{dl})$ & $26,71 \pm 8,80$ & $39,40 \pm 14,89$ & 0,000 \\
TG $(\mathrm{mg} / \mathrm{dl})$ & $131,51 \pm 31,36$ & $163,30 \pm 42,91$ & 0,000 \\
\hline CT/HDL-C & $4,68 \pm 1,02$ & $4,53 \pm 1,13$ & $\mathrm{~ns}$ \\
LDL-C/HDL-C & $2,62 \pm 0,86$ & $2,88 \pm 0,71$ & 0,002 \\
\hline
\end{tabular}


Tabela 2. Perfil lipídico de acordo com a prática habitual de atividade física ajustado para idade e IMC.

\begin{tabular}{|c|c|c|c|c|c|}
\hline \multicolumn{6}{|c|}{ Prática Habitual de Atividade Física } \\
\hline & Muito Ativo & Ativo & Irregularmente Ativo & Sedentário & $\mathbf{p}$ \\
\hline \multicolumn{6}{|l|}{ Mulheres } \\
\hline $\mathrm{n}$ & 23 & 29 & 77 & 67 & \\
\hline $\mathrm{CT}(\mathrm{mg} / \mathrm{dl})$ & $181,00 \pm 32,66$ & $186,33 \pm 39,23$ & $221,06 \pm 39,89$ & $227,44 \pm 26,74$ & 0,001 \\
\hline $\mathrm{HDL}-\mathrm{C}(\mathrm{mg} / \mathrm{dl})$ & $53,25 \pm 17,22$ & $53,82 \pm 12,58$ & $45,85 \pm 17,41$ & $47,94 \pm 9,01$ & ns \\
\hline LDL-C (mg/dl) & $99,75 \pm 37,63$ & $121,73 \pm 35,75$ & $143,40 \pm 35,87$ & $148,68 \pm 21,81$ & 0,000 \\
\hline VLDL-C (mg/dl) & $25,63 \pm 9,91$ & $23,83 \pm 9,40$ & $25,39 \pm 9,86$ & $30,83 \pm 9,23$ & ns \\
\hline TG (mg/dl) & $115,64 \pm 41,90$ & $127,00 \pm 49,26$ & $123,13 \pm 68,71$ & $154,19 \pm 71,12$ & 0,020 \\
\hline $\mathrm{CT} / \mathrm{HDL}-\mathrm{C}$ & $3,67 \pm 0,81$ & $3,47 \pm 1,10$ & $5,52 \pm 1,85$ & $4,92 \pm 1,17$ & 0,038 \\
\hline LDL-C/HDL-C & $2,02 \pm 0,87$ & $2,06 \pm 1,03$ & $3,71 \pm 1,04$ & $3,24 \pm 0,91$ & 0,001 \\
\hline \multicolumn{6}{|l|}{ Homens } \\
\hline $\mathrm{n}$ & 26 & 31 & 55 & 53 & \\
\hline $\mathrm{CT}(\mathrm{mg} / \mathrm{dl})$ & $158,86 \pm 14,25$ & $195,50 \pm 39,68$ & $203,12 \pm 34,67$ & $211,08 \pm 28,59$ & 0,001 \\
\hline HDL-C (mg/dl) & $41,00 \pm 11,93$ & $38,50 \pm 6,93$ & $40,59 \pm 10,34$ & $37,50 \pm 8,27$ & ns \\
\hline LDL-C (mg/dl) & $88,57 \pm 16,57$ & $106,78 \pm 31,28$ & $128,32 \pm 40,79$ & $138,68 \pm 33,42$ & 0,004 \\
\hline VLDL-C (mg/dl) & $35,17 \pm 14,70$ & $37,20 \pm 16,05$ & $34,90 \pm 13,54$ & $40,06 \pm 13,12$ & ns \\
\hline $\mathrm{TG}(\mathrm{mg} / \mathrm{dl})$ & $146,14 \pm 35,06$ & $144,38 \pm 29,08$ & $171,00 \pm 37,20$ & $175,00 \pm 37,93$ & 0,043 \\
\hline CT/HDL-C & $4,14 \pm 1,16$ & $5,32 \pm 2,11$ & $4,98 \pm 1,38$ & $5,93 \pm 1,61$ & 0,017 \\
\hline LDL-C/HDL-C & $2,26 \pm 0,49$ & $3,50 \pm 1,48$ & $2,75 \pm 1,24$ & $3,95 \pm 1,39$ & 0,007 \\
\hline
\end{tabular}

das ativas em relação às irregularmente ativas e sedentárias. Em ambos os sexos as diferenças observadas entre os estratos dos muito-ativos e dos ativos não são apontadas em linguagem estatística.

Quanto às proporções de sujeitos categorizados nos quatro estratos de prática habitual de atividade física preconizados pelo IPAQ, verifica-se que a distribuição dos valores é bastante similar em ambos os sexos; no entanto, a proporção de sujeitos categorizados conjuntamente como sedentário e irregularmente ativo $(73,5 \%$ das mulheres e $65,4 \%$ dos homens) é acentuadamente mais elevada que a proporção de sujeitos que atendem as recomendações propostas para os ativos e os muitoativos (26,5\% das mulheres e $34,6 \%$ dos homens).

A tabela 3 mostra as dimensões de OR acompanhados dos intervalos de confiança equivalentes ao risco relativo de os sujeitos analisados no estudo apresentarem perfil lipídico de risco aterogênico em função da categorização em estratos de prática habitual de atividade física. Os resultados da análise de regressão logística indicam que as dimensões das ORs para dislipidemias são mais elevadas entre os sujeitos de menor prática habitual de atividade física. Na presente casuística, homens categorizados como sedentários, mediante os critérios propostos pelo IPAQ, demonstram risco relativo significativamente mais elevado de apresentarem teores de CT $(\mathrm{OR}=1,52 ; 95 \%$ IC 1,14-1,98) e de TG $(\mathrm{OR}=1,37 ; 95 \%$ IC 1,03-1,78) comprometedores em comparação com seus pares muito-ativos. No caso das mulheres, aquelas que relataram ser sedentárias demonstram possuir de 38\% (LDL-C - OR= 1,38; 95\% IC $1,02-1,84)$ a $59 \%(\mathrm{TG}-\mathrm{OR}=1,59 ; 95 \%$ IC 1,15-2,08) mais chances de apresentarem perfil aterogênico de lipídeos plasmáticos se comparadas com aquelas que relataram ser muito-ativas.

\section{DISCUSSÃo}

Em razão da facilidade de aplicação em grandes grupos, do menor custo e por permitir coletar informações com relação ao tipo e ao contexto em que são realizados, o que não ocorre com os demais recursos de medida, os questionários representam o instrumento mais acessível para a avaliação da prática habitual de atividade física, sobretudo em estudos de natureza epidemiológica. Em sendo assim, encontra-se disponível na literatura uma grande variedade de opções de questionários que procuram contemplar diferentes domínios da atividade física. Os primeiros questionários sugeridos procuravam enfocar as atividades físicas ocupacionais $(24,25)$. Na seqüência, a ênfase dos questionários se dirigiu para as atividades físicas no tempo livre $(9,10)$. Recentemente, tem-se reconhecido a importância de outros tipos de atividades físicas que os sujeitos realizam como parte de seu cotidiano, como trabalho doméstico, caminhar e andar de bicicleta como meio de transporte (17). 
As múltiplas abordagens quanto aos domínios associados à prática habitual de atividade física têm contribuído para que os critérios de avaliação possam se diferenciar consideravelmente entre as diferentes opções de questionários disponibilizadas. Esta situação tem limitado, na maioria dos casos, comparações mais seguras entre segmentos de uma mesma população ou entre sujeitos pertencentes a diferentes populações quanto à prevalência de diferentes estratos de prática habitual de atividade física. Como resposta a este desafio, um grupo de especialistas iniciou em 1998 intensa discussão direcionada à proposição de um questionário internacional que pudesse ser empregado como instrumento comum com finalidade de levantar dados nacionais e internacionais comparáveis da atividade física. Como produto deste esforço foi idealizado o IPAQ, atualmente validado em 12 países, inclusive no Brasil $(22,26)$. Os resultados destas validações sugerem que o IPAQ apresenta aceitáveis propriedades de medida para ser empregado em diferentes contextos, e que torna-se apropriado para estudos populacionais de prevalência quanto à prática habitual de atividade física. Baseando-se nessas evidências, mais recentemente, inúmeros pesquisadores têm procurado desenvolver seus estudos envolvendo o IPAQ como instrumento de medida associado à prática habitual de atividade física $(23,27,28)$.

Vários estudos prévios disponibilizados na literatura procuram relatar a associação entre a prática de atividade física e as concentrações plasmáticas de lipídeos (1-4). No entanto, para o nosso conhecimento, esta parece ser a primeira experiência publicada em que se procurou analisar o impacto da prática habitual de atividade física e o perfil lipídico, envolvendo o IPAQ como instrumento de medida.

Diferenças entre ambos os sexos quanto aos valores médios de concentrações plasmáticas de lipídeos observadas no estudo coincidem com tendências encontradas na literatura (2-4). Indicações encontradas em publicações anteriores apontam que o dimorfismo sexual observado nos níveis de lipídeos plasmáticos pode ser explicado em razão do perfil hormonal de cada sexo, apontando um padrão lipídico de maior risco aterogênico entre os homens frente às mulheres (5-7).

Consistente com resultados de outros estudos, também envolvendo o IPAQ como instrumento de medida relacionado à prática habitual de atividade física $(23,27,28)$, os resultados encontrados apontam os homens como sendo discretamente mais ativos fisicamente. Mediante idênticos critérios de interpretação dos dados coletados, os resultados apresentados pelos sujeitos aqui analisados apontam uma proporção mais elevada de sedentarismo em comparação com estudos europeus (27); porém, proporções similares, se comparados com estudos envolvendo segmentos da população brasileira (23) e de países sul-americanos (28). As razões para essas diferenças não são totalmente compreendidas. Em parte, variações demográficas sazonais nos níveis habituais de prática de atividade física podem contribuir para uma menor proporção de sujeitos ativos no presente estudo. Adicionalmente, diferenças metodológicas na seleção das amostras entre os dois estudos podem ter produzido as diferenças observadas.

O principal achado do presente estudo revelou que níveis mais elevados de prática habitual de atividade física estão associados aos valores de lipídeos plasmáticos de menor magnitude. Além do que, o gradiente entre a prática habitual de atividade física e os teores de lipídeos plasmáticos desfavoráveis apresentou comportamento específico em cada sexo. Os valores de OR também indicaram risco mais elevado de se encontrar perfil lipídico de risco aterogênico em grupos de sujeitos sedentários comparados com seus pares fisicamente mais ativos. Confirmando expectativas encontradas na literatura, esses resultados são consistentes com estudos realizados anteriormente envolvendo outros instrumentos de medida direcionados à monitoração da atividade física (1-4).

Mecanismos fisiológicos baseados na prática de atividade física induzem a um perfil lipídico favorável resultante de complexas interações envolvendo hormônios, enzimas e receptores. Estudos sugerem que o aumento na atividade da lipoproteína lipase no músculo esquelético e no tecido adiposo durante a realização de esforços físicos de intensidades moderada-a-vigorosa, e por algum tempo pós-esforço, associado ao possível decréscimo da síntese hepática dos TG, possa ser ajustes metabólicos que favorecem as menores concentrações de lipídeos plasmáticos entre os sujeitos mais ativos fisicamente $(29,30)$.

Por vezes, especula-se que as modificações favoráveis no perfil lipídico induzidas pela prática da atividade física possam confundir-se com concomitantes alterações no peso corporal, por conta do maior dispêndio energético proveniente dos esforços físicos realizados (31). Neste particular, no presente estudo os valores de IMC, juntamente com a idade dos sujeitos, foram controlados estatisticamente mediante a análise de co-variância. Mesmo na ausência de participação dos valores de IMC e da idade, e confirmando resultados de importantes estudos envolvendo delineamentos experimentais bem elaborados $(32,33)$, os resultados encontrados no presente estudo mostraram 
que os sujeitos pertencentes ao estrato dos mais ativos fisicamente apresentaram perfil lipídico mais favorável.

Similar ao que foi observado em estudos anteriores $(2,3,5)$, os resultados encontrados no presente estudo revelaram importantes diferenças entre ambos os sexos quanto ao gradiente da prática habitual de atividade física e dos teores de lipídeos plasmáticos desfavoráveis. Enquanto nos homens as diferenças mais acentuadas foram constatadas entre o estrato dos muito-ativos em comparação com seus pares irregularmente ativos e sedentários, nas mulheres esse fenômeno foi observado entre o estrato das ativas em relação às irregularmente ativas e sedentárias. Além de eventuais limitações atribuídas à composição da amostra e ao não-controle de possíveis variáveis de confundimento, sobretudo aquelas vinculadas ao campo dietético, justificativa plausível para o fato, talvez possa estar alicerçada em fatores hormonais específicos de cada sexo, que induzem aos diferentes graus de interação atividade física-perfil lipídico.

Os valores de OR encontrados comprovaram que o comportamento e a extensão das associações entre a prática habitual de atividade física e o perfil lipídico apresentam magnitudes diferentes em cada sexo. Enquanto entre os homens o sedentarismo se associou mais intensamente com o CT, entre as mulheres a prática insuficiente de atividade física desempenhou papel mais importante nas maiores concentrações de TG. Esses achados coincidem com resultados de estudos de intervenção que, ao elevar a prática de atividade física mediante envolvimento em programas sistematizados de exercícios físicos, comprovaram diminuição mais acentuada nas concentrações de CT e TG entre sujeitos adultos (29).

As menores dimensões de OR observadas entre os estratos de prática habitual de atividade física e as concentrações desfavoráveis de HDL-C são consistentes com as fracas associações encontradas em outros estudos $(2,3)$, e confirmam evidências no sentido de que a prática insuficiente de atividade física pode eventualmente apresentar associações de menor extensão com um perfil plasmático aterogênico que a ingestão excessiva de gorduras total e saturada $(34,35)$ e o uso de tabaco (36).

Em conclusão, existem similaridades entre os achados do presente estudo e os resultados prévios provenientes de experimentos controlados, envolvendo informações quanto ao nível de prática habitual de atividade física e ao perfil lipídico. No entanto, atentase para o fato de que, diferentemente dos demais, no presente estudo optou-se por acompanhar o nível de prática habitual de atividade física por intermédio do
IPAQ. Portanto, existem fortes indicações no sentido de que o IPAQ possa se definir como um adequado instrumento direcionado ao acompanhamento dos riscos aterogênicos vinculados à prática habitual inadequada de atividade física.

\section{REFERÊNCIAS}

1. Katzmarzyk PT, Church TS, Blair SN. Cardiorespiratory fitness attenuates the effects of the metabolic syndrome on all-cause and cardiovascular disease mortality in men. Arch Intern Med 2004:164:1092-7.

2. Forrest $\mathrm{KY}$, Bunker $\mathrm{CH}$, Kriska AM, Ukoli FA, Huston SL, Markovi CN. Physical activity and cardiovascular risk factors in a developing population. Med Sci Sports Exerc 2001;33:1598-604.

3. MacAuley D, McCrum EE, Stott G, Evans AE, Duly E, Trinick $T R$, et al. Physical activity, lipids, apolipoproteins, and Lp(a) in the Northern Ireland Health and Activity Survey. Med Sci Sports Exerc 1996;26:720-36.

4. Guedes DP, Guedes JERP. Atividade física, aptidão cardiorrespiratória, composição da dieta e fatores de risco predisponentes às doenças cardiovasculares. Arq Bras Cardiol 2001;77:243-50.

5. Eaton CB, Lapane KL, Garber CE, Assaf AR, Lasater TM, Carleton RA. Physical activity, physical fitness, and coronary heart disease risk factors. Med Sci Sports Exerc 1995;27:340-6.

6. Berlin JA, Colditz A. A meta-analysis of physical activity in the prevention of coronary heart disease. Am J Epidemiol 1990;132:612-27.

7. Bassuk SS, Manson JE. Physical activity and cardiovascular disease prevention in women: how much is good enough? Exerc Sport Sci Rev 2003;31:176-81.

8. Powel KE, Thompson PD, Caspersen CJ. Physical activity and the incidence of coronary heart disease. Annu Rev Public Health 1987;8:253-87.

9. Leon AS, Connett J, Jacobs DR Jr, Rauramaa R. Leisure-time physical activity levels and risk of coronary heart disease and dealt. The Multiple Risk Factor Intervention Trial. JAMA 1987;258:2388-95.

10. Helmer U, Herman B, Shea S. Moderate and vigorous leisuretime physical activity and cardiovascular disease risk factors in West Germany, 1984-1991. Int J Epidemiol 1994;23:28592.

11. Paffenbarger RS Jr, Blair SN, Lee IM. A history of physical activity, cardiovascular health and longevity: the scientific contributions of Jeremy N Morris, DSc. DPH, FRCP. Int J Epidemiol 2001;30:1184-92.

12. Després JP, Lamarche B. Low-intensity endurance exercise training, plasma lipoproteins and the risk of coronary heart disease. J Intern Med 1994;236:7-22.

13. Marrugat J, Elosua R, Covas MI, Molina L, Rubiés-Prat J. Amount and intensity of physical activity, physical fitness and serum lipids in men. Am J Epidemiol 1996;143:562-9.

14. Fung TT, Hu FB, Yu J, Chu NF, Spiegelman D, Tofler GH, et al. Leisure-time physical activity, television watching, and plasma biomarkers of obesity and cardiovascular risk. Am J Epidemiol 2000;152:1171-8.

15. Miller YD, Dunstan DW. The effectiveness of physical activity interventions for the treatment of overweight and obesity and type 2 diabetes. J Sci Med Sport 2004;7(suppl 1):52-9.

16. Montoye HJ, Kemper HCG, Saris WHM, Washburn RA. Measuring Physical Activity and Energy Expenditure. Champaign: Human Kinetics, 1996.

17. Kriska AM, Caspersen CJ. A collection of physical activity questionnaires for health-related research. Med Sci Sports Exerc 1997;29:S1-S205. 
18. Sallis JF, Saelens BE. Assessment of physical activity by selfreports: status, limitations, and future directions. Res $\mathbf{Q}$ Exerc Sport 2000;71:1-14.

19. Gordon CC, Chumlea WC, Roche AP. Stature, recumbent length, and weight. In Lohman TG, Roche AP, Martorell R (ed.). Anthropometric Standardization Reference Manual. Champaign: Human Kinetics, 1988. pp. 3-8.

20. Friedewald WT, Levy RI, Frederickson DS. Estimation of the concentration of low-density lipoprotein cholesterol in plasma, without use of preparative ultracentrifuge. Clin Chem 1972;18:499-502.

21. Sociedade Brasileira de Cardiologia. III Diretrizes Brasileiras sobre Dislipidemias e Diretrizes de Prevenção da Aterosclerose do Departamento de Aterosclerose da Sociedade Brasileira de Cardiologia. Arq Bras Cardiol 2001;77(supl. 3).

22. Craig $C L$, Marshall $A L$, Sjöström $M$, Bauman $A E$, Booth $M L$, Ainsworth BE, et al. International Physical Activity Questionnaire: 12-Countr reliability and validity. Med Sci Sports Exerc 2003:35:1381-95.

23. Matsudo SM, Matsudo VR, Araújo T, Andrade D, Andrade E, Oliveira $L$, et al. Nível de atividade física da população do estado de São Paulo: análise de acordo com o gênero, idade, nível socioeconômico, distribuição geográfica e de conhecimento. Rev Bras Cien Mov 2000;10:41-50.

24. Morris JN, Healdy HA, Raffle PA, Roberts CG, Parks JW. Coronary heart disease and physical activity of work. Lancet 1953;265:1053-7.

25. Paffenbarger RS, Hale WE. Work activity and coronary heart mortality. N Engl J Med 1975;292:545-50.

26. Matsudo SM, Araújo T, Matsudo VR. Questionário Internacional de Atividade Física (IPAQ): estudo de validade e reprodutibilidade no Brasil. Rev Bras At Física \& Saúde 2001;6:5-18.

27. Rütten A, Abu-Omar K. Prevalence of physical activity in the European Union. Soz Präventivmed 2004;49:281-9.

28. Gómez LF, Duperly J, Lucumi DI, Gómez R, Venegas AS. Nivel de actividad física global en la población adulta de Bogotá (Colombia). Prevalencia y factores asociados. Gac Sanit 2005;19:206-13.
29. Durstine JL, Grandjean PW, Davis PG, Ferguson MA, Alderson $\mathrm{NI}$, et al. Blood lipid and lipoprotein adaptations to exercise: a quantitative analysis. Sports Med 2001;31:1033-62.

30. Williams PT. Health effects resulting from exercise versus those from body fat loss. Med Sci Sports Exerc 2001;33:S611-21.

31. Després JP, Lamarche B, Bouchard C. Exercise and the prevention of dyslipidemia and coronary heart disease. Int $\mathbf{J}$ Obes 1995; 19(suppl 4):S45-S51.

32. Katzel LI, Bleecker ER, Colman EG. Effects of weight loss vs. aerobic exercise training on risk factors for coronary disease in healthy, obese, middle-aged and older men. JAMA 1995;274:1915-21.

33. Lamarche B, Després JP, Pouliot MC. Is body fat loss a determinant factor in the improvement of carbohydrate and lipid metabolism following aerobic exercise training in obese women? Metabolism 1992;41:1249-56.

34. Porrini M, Simonetti P, Testolin G, Roggi C, Laddomada MS, Tenconi MT. Relation between diet composition and coronary heart disease risk factors. J Epidemiol Comm Health 1991;45:148-51.

35. Esrey KL, Joseph L, Grover SA. Relationship between dietary intake and coronary heart disease mortality: Lipid Research Clinics Prevalence Follow-up Study. J Clin Epidemiol 1996;49:211-6.

36. Price JF, Mowbray PI, Lee AJ, Rumley A, Lowe GD, Fowkes FGR. Relationship between smoking and cardiovascular risk factors in the development of peripheral arterial disease and coronary artery disease. Edinburgh Artery Study. Eur Heart J 1999;20:344-53.

\section{Endereço para correspondência:}

Dartagnan Pinto Guedes

Rua Ildefonso Werner 177

Condomínio Royal Golf

86055-545 Londrina, PR

E-mail: darta@sercomtel.com.br 